\title{
Downregulation of BRD4 inhibits gallbladder cancer proliferation and metastasis and induces apoptosis via PI3K/AKT pathway
}

\author{
JIAQI HAO ${ }^{1,2^{*}}$, ZIYI YANG ${ }^{1,2^{*}}$, LEI WANG ${ }^{3}$, YIJIAN ZHANG ${ }^{1,2}$, YIJUN SHU $^{1,2}$, \\ LIN JIANG $^{1,2}$, YUNPING HU ${ }^{1,2}$, WENJIE LV ${ }^{1,2}$, PING DONG ${ }^{1,2}$ and YINGBIN LIU ${ }^{1,2}$ \\ ${ }^{1}$ Department of General Surgery, Xinhua Hospital Affiliated to Shanghai Jiao Tong University \\ School of Medicine; ${ }^{2}$ The Institute of Biliary Disease Research, School of Medicine, \\ Shanghai Jiao Tong University, Shanghai; ${ }^{3}$ Department of Hepatobiliary Surgery, \\ Nanjing Medical University Affiliated Wuxi Second Hospital, Wuxi, Jiangsu, P.R. China
}

Received March 6, 2017; Accepted July 7, 2017

DOI: 10.3892/ijo.2017.4081

\begin{abstract}
Bromodomain containing protein 4 (BRD4) has been demonstrated to play a critical role in tumor progression. However, the expression and function of BRD4 in gallbladder cancer (GBC) are still unknown. In this study, we report that BRD4 expression level was significantly upregulated in GBC tissues and GBC cell lines. We explored the correlation between BRD4 levels and clinicopathological data of GBC patients. The high expression level of BRD4 was notably correlated with the poor prognosis of GBC patients. Knockdown of BRD4 suppressed proliferation and migration in NOZ and EH-GB1 cells. The depletion of BRD4 in GBC cell lines resulted in obvious cell apoptosis and downregulated the expression levels of Bcl-2, p-PI3K and p-AKT. In vivo, tumor volumes of nude mice were significantly decreased in BRD4 silenced group. Our data suggested that downregulation of BRD4 in GBC cells induced apoptosis by PI3K/AKT pathway. Inhibition of BRD4 expression may be a novel therapeutic strategy for patients with GBC.
\end{abstract}

\section{Introduction}

Gallbladder cancer (GBC) is the most common and aggressive cancer of the biliary tract, it constitutes $80-85 \%$ of total biliary tract cancer. GBC shows a striking geographical variation in its incidence, with high incidence has been reported from

Correspondence to: Dr Ping Dong or Professor Yingbin Liu, Department of General Surgery, Xinhua Hospital Affiliated to Shanghai Jiao Tong University School of Medicine, 1665 Kongjiang Road, Shanghai 200092, P.R. China

E-mail: dongping1050@163.com

E-mail: liuybphd@126.com

${ }^{*}$ Contributed equally

Key words: BRD4, gallbladder cancer, tumor progression, apoptosis, PI3K/AKT pathway
Asia, South America and some part of central Europe (1). GBC incidence is relatively low $(2.2 / 100,000)$ (2). However, GBC is a notoriously lethal disease and has a poor prognosis, the mean overall survival time is 6 months and 5-year survival rate is $5 \%$ (3). Thus, it is critical to investigate the molecular mechanisms of GBC progression, which may be helpful to identify novel diagnostic and therapeutic target.

The bromodomain and extra terminal (BET) proteins, which include BRD2, BRD3, BRD4 and BRDT, regulate gene expression as the acetylated lysine binding domain in chromatin $(4,5)$. As the best-studied member of the BET family, Bromodomain containing protein 4 (BRD4) has been demonstrated to play an essential role in cell cycle and cell proliferation $(6,7)$. BRD4 functions as a transcription regulator through interactions with P-TEFb, MYC and NF- $\mathrm{B}(8,9)$. Deregulation of BRD4 protein is increasingly found in several different types of cancers such as colorectal cancer, breast cancer, pancreatic cancer and lung cancer (5,10-12). BRD4 is considered to be a compelling therapeutic target. However, the role of BRD4 has not been well studied for gallbladder cancer.

In this study, we investigated the expression level and potential roles of BRD4 in GBC, aiming to provide novel insight into GBC diagnosis and therapy.

\section{Materials and methods}

Patient samples. All specimens were achieved from $51 \mathrm{GBC}$ patients with pathologically confirmed GBC after surgery between 2013 and 2014 in Xinhua Hospital Affiliated to Shanghai Jiaotong University. None of the patients received radiotherapy or chemotherapy before surgery. The tissue samples were staged on the basis of the AGCC-TNM Classification of Malignant Tumors ( 7 th edition). The pathological classification was reviewed separately by two experienced pathologists. Tissue samples were embedded in paraffin prior to immunohistochemistry analysis. For quantitative real-time PCR, part of specimens were immediately frozen in liquid nitrogen after resection. Informed consent was obtained in all cases. Ethical approval was obtained from the ethics committee of the Xinhua Hospital Affiliated to Shanghai Jiao Tong University School of Medicine. 
Immunohistochemistry. Immunohistochemistry (IHC) staining was performed according to the standard immunoperoxidase staining procedure (13). Immunoreactivity was scored as follows: $0,<5 \%$ immunoreactive cells; $1,5-25 \%$ immunoreactive cells; 2, 25-50\% immunoreactive cells; $3,50-75 \%$ immunoreactive cells and 4 for $>50 \%$ immunoreactive cells. The staining intensity of BRD4 expression was visually scored as follows: 0 , negative staining; 1 , weak staining; 2 , moderate staining and 3 for strong staining. The final score was the sum of extent and intensity. The specimens were classified as negative (0-1), weak (2-3), moderate (4-5), and strong (6-7) staining.

Quantitative real-time PCR. Total RNA was extracted from GBC tissues and cells using TRIzol reagent. cDNA was synthesized by using PrimeScript Reverse Transcriptase (Takara, Osaka, Japan) following the manufacturer's instructions. The expression level of BRD4 was measured using the SYBR-Green method, and products were detected by StepOnePlus ${ }^{\mathrm{TM}}$ Real-time PCR system (Applied Biosystems, Foster City, CA, USA). The primers used for amplification of BRD4: BRD4 (forward primer) 5'-ACAACAAGCCTGGAG ATGACA-3', BRD4 (reverse primer) 5'-GTTTGGTACCGT GGAAACGC-3'; GAPDH (forward primer) 5'-CAACAGCC TCAAGATCATCAGC-3', GAPDH (reverse primer) 5'-TTCTA GACGGCAGGTCAGGTC-3'. The relative gene expression levels were calculated using the $2^{-\Delta \Delta \mathrm{CT}}$ analysis method (14).

Cell culture. The human GBC cell lines NOZ, EH-GB1, GBC-SD, SGC-996 and OCUG were obtained from the Shanghai Cell Institute National Cell Bank. NOZ and SGC-996 were cultured in Williams' medium E and RPMI-1640 medium (HyClone, Logan, UT, USA), respectively. The EH-GB1, GBC-SD and OCUG cells were cultured in DMEM (Gibco, Grand Island, NY, USA). Cells were supplemented with $15 \%$ fetal bovine serum (FBS; Gibco) at $37^{\circ} \mathrm{C}$ in a $5 \%$ $\mathrm{CO}_{2}$ incubator.

Immunofluorescence analysis. Cells were fixed in $4 \%$ paraformaldehyde for $15 \mathrm{~min}$ at room temperature and then permeabilized in $0.5 \%$ Triton for $5 \mathrm{~min} .3 \% \mathrm{BSA}$ was used for blocking. The cells were incubated with anti-BRD4 antibody at $4^{\circ} \mathrm{C}$ for $12 \mathrm{~h}$. Then, the cells were incubated with goat anti-rabbit IgG (Beyotime, Shanghai, China) for $30 \mathrm{~min}$, and counterstained with Hoechst 33342 (Beyotime). A fluorescence microscope (Leica DMI3000B; Leica, Wetzlar, Germany) was used for observation.

siRNA, plasmid and Lv-shRNA transfection. BRD4 siRNAs were designed and synthesized by Biotend (Shanghai, China). The siRNAs targeting human BRD4 were: BRD4-siRNA1: 5'-CCUGAUUACUAUAAGAUCAdTdT-3', BRD4-siRNA2: 5'-CAGUGACAGUUCGACUGAUdTdT-3'. Cells were plated onto 6-well plates at $30 \%$ density and transfected with BRD4-siRNA or NC-siRNA using Lipofectamine 2000 (Invitrogen, Carlsbad, CA, USA) according to the manufacturer's protocol.

The pcDNA4c hBrd4 was obtained from Addgene (Plasmid 14441), and the amino acid change T249P was modified by Longqian Biotech. Viafect Transfection Reagent was
Table I. Relationship between BRD4 expression and relevant factors in GBC patients.

\begin{tabular}{|c|c|c|c|c|}
\hline \multirow[b]{2}{*}{ Factors } & \multirow[b]{2}{*}{ Cases } & \multicolumn{2}{|c|}{ BRD4 expression } & \multirow[b]{2}{*}{ P-value } \\
\hline & & Low & High & \\
\hline Sex & & & & 0.727 \\
\hline Male & 10 & 4 & 6 & \\
\hline Female & 41 & 14 & 27 & \\
\hline Age (years) & & & & 0.028 \\
\hline$<60$ & 10 & 7 & 3 & \\
\hline$\geq 60$ & 41 & 11 & 30 & \\
\hline Histology & & & & 0.574 \\
\hline Well/moderate & 45 & 17 & 28 & \\
\hline Poor & 6 & 1 & 5 & \\
\hline $\mathrm{T}$ & & & & $<0.001$ \\
\hline Tis+T1+T2 & 20 & 14 & 6 & \\
\hline $\mathrm{T} 3+\mathrm{T} 4$ & 31 & 4 & 27 & \\
\hline $\mathrm{N}$ & & & & 0.025 \\
\hline Absent & 26 & 13 & 13 & \\
\hline Present & 25 & 5 & 20 & \\
\hline M & & & & 0.141 \\
\hline Negative & 45 & 18 & 27 & \\
\hline Positive & 6 & 0 & 6 & \\
\hline Tumor stage & & & & 0.002 \\
\hline $0-\mathrm{I}$ & 6 & 6 & 0 & \\
\hline II-IV & 45 & 12 & 33 & \\
\hline
\end{tabular}

used for transfection. BRD4 expression level was detected by qRT-PCR and western blotting.

pGMLV-SC5 RNAi vectors encoding BRD4 (5'-CCTGAT TACTATAAGATCA-3') were constructed by Genomeditech (Shanghai, China). NOZ cells were infected at a multiplicity of infection (MOI) of 80 in complete culture medium for $24 \mathrm{~h}$. qRT-PCR and western blotting were used to examine the knockdown efficiency.

In vitro cell proliferation assays. The CCK-8 (Cell Counting Kit-8; Dojindo, Kumamoto, Japan) assay was performed to evaluate cell proliferation. NOZ and EH-GB1 cells were plated at a density of $1 \times 10^{3}$ cells per well in 96 -well plates. The absorbance value (OD) was measured at $450 \mathrm{~nm}$ using a Spectra Max 190 (Molecular Devices, Sunnyvale, CA, USA).

In the colony formation assay, cells were planted in 6-well plates with 600 cells per well and were cultured for 9 and 14 days, respectively. Then, the colonies were fixed in $4 \%$ paraformaldehyde and stained with $0.5 \%$ crystal violet (Beyotime). The number of colonies was photographed and counted under a microscope.

Migration and invasion assays. Cell migration and invasion were performed using 24-well Transwell chambers (Corning Inc., Corning, NY, USA). Briefly, the upper chamber was seeded 
A

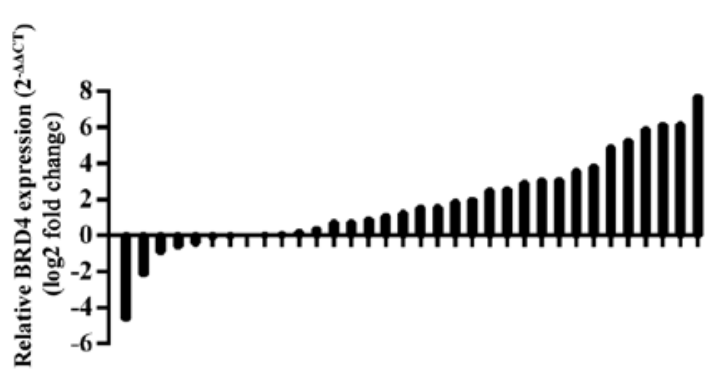

B

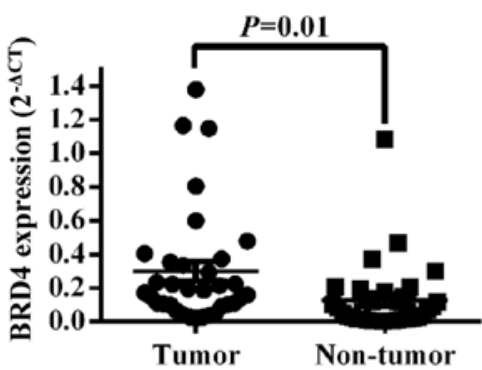

C

Gallbaldder cancer

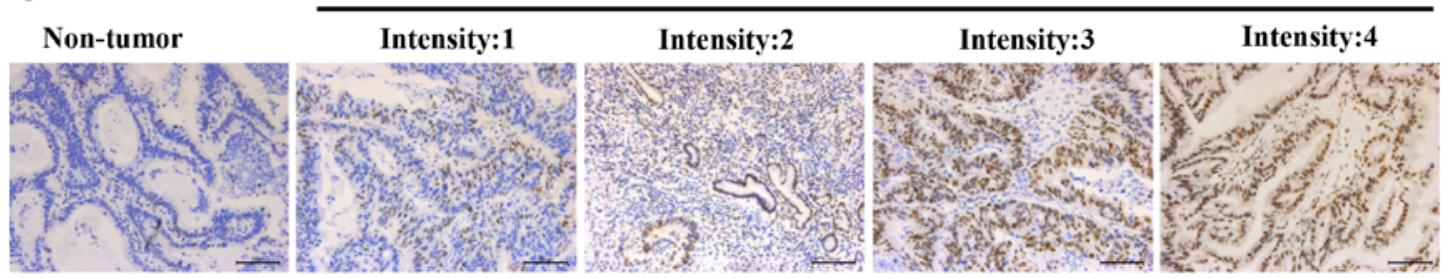

D

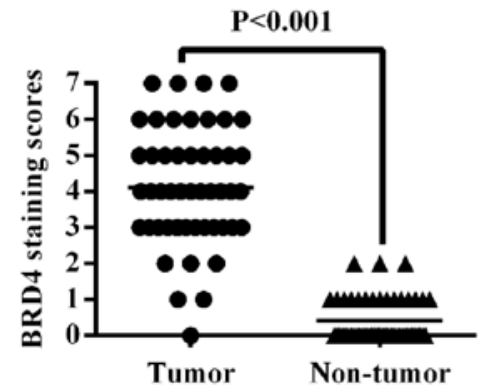

E

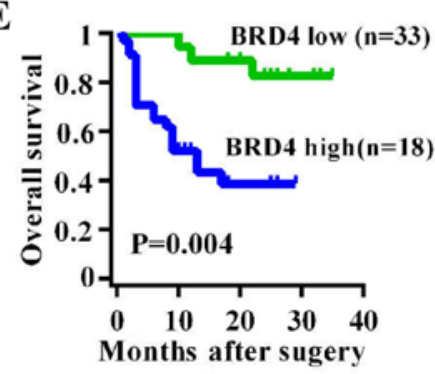

Figure 1. BRD4 overexpression is associated with poor prognosis of GBC patients. (A) The comparison of BRD4 mRNA expression level between GBC tumor tissues and adjacent non-tumor tissues using qRT-PCR. (B) Relative BRD4 expression level in GBC tissues and their corresponding non-tumor tissues (2--ACT). (C) IHC staining for BRD4 expression in non-tumor tissues and tumor tissues from GBC patients (scale bar, $100 \mu \mathrm{m}$ ). (D) Average staining scores for BRD4 expression in GBC tumor tissues and adjacent tissues $(\mathrm{P}<0.001)$. (E) The overall survival of GBC patients based on BRD4 expression level.

with $2 \times 10^{4}$ cells in $200 \mu 1$ serum-free medium, while the lower chamber was filled with $500 \mu \mathrm{l}$ basal medium containing $15 \%$ FBS. After $20 \mathrm{~h}$ of incubation, cells were fixed with $4 \%$ paraformaldehyde for $20 \mathrm{~min}$ and stained with crystal violet for $20 \mathrm{~min}$. Then cells on the upper surface of the upper chambers were scraped. Five fields were chosen randomly for analysis.

Apoptosis assay. Apoptosis was inspected using an Annexin V-FITC Apoptosis Detection kit (BD Pharmingen, San Diego, CA, USA). Cells were harvested and washed three times with PBS. Next, $100 \mu 1$ of Annexin V binding buffer (1X), $5 \mu \mathrm{l}$ of PI $(50 \mu \mathrm{g} / \mathrm{ml})$ and $5 \mu \mathrm{l}$ of FITC (BD Pharmingen) were added to the cell suspensions and incubated for $15 \mathrm{~min}$ at room temperature in the dark. Apoptosis array was analyzed using FACS Canto II (BD Pharmingen).

Western blotting. Total protein was extracted using RIPA buffer (Cell Signaling, Danvers, MA, USA). Cell proteins were separated by SDS-PAGE and then transferred onto PVDF membranes (Millipore Corp., Billerica, MA, USA). Blots were blocked with 5\% skim milk at room temperature for $2 \mathrm{~h}$. The PVDF membranes were reacted with a series of primary antibodies at $4^{\circ} \mathrm{C}$ overnight. The anti-BRD4 antibody was acquired from Abcam (Cambridge, MA, USA). Primary antibodies against E-cadherin, vimentin, N-cadherin, Bax, Bad, Bcl-2, PI3K, p-PI3K, AKT, p-AKT, JNK, p-JNK, GAPDH were obtained from Cell Signaling Technology. Then, the blots were reacted with the appropriate HRP-conjugated secondary antibody and quantified using a Gel Doc 2000 (Bio-Rad, Hercules, CA, USA).

Inhibitor reagent. PI3K/AKT pathway inhibitor GDC-0941 was purchased from MCE. Cells were treated with different GDC-0941 concentrations of $0,1,3,10 \mu \mathrm{M}$. Total proteins were isolated for western blotting after $72 \mathrm{~h}$ incubation.

Nude mouse subcutaneous xenograft model. Male nude mice (four-week-old) were purchased from the Shanghai Laboratory Animal Center of the Chinese Academy of Sciences (Shanghai, China). All procedures were approved by the Ethics Committee of Xinhua Hospital Affiliated to Shanghai Jiaotong University School of Medicine. NOZ cells (Lv-shNC/Lv-shBRD4 group, $2 \times 10^{6}$ ) were subcutaneously injected into the left axilla of nude mice ( $n=5$ mice/group). The tumour volumes were measured $\left(1 / 2 \times\right.$ width $^{2} \mathrm{x}$ length) weekly. The nude mice were sacrificed after 4 weeks and tumors were weighed. Tumor tissues were stored for western blotting and qRT-PCR analysis.

Statistical analysis. Statistical analyses were performed with SPSS software (version 22.0). The Student's t-test and nonparametric tests were used when necessary. The Kaplan-Meier test was used for the overall survival analysis. Subsequently, 

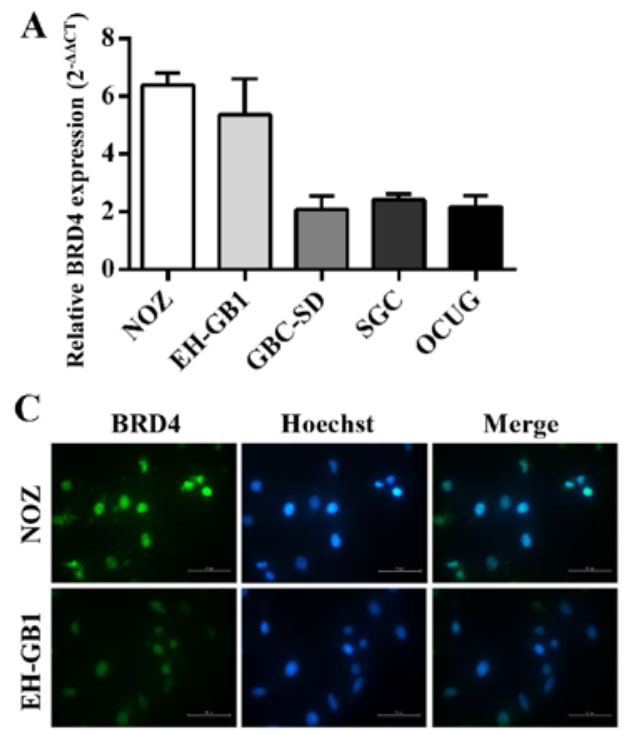

$\mathbf{E}$

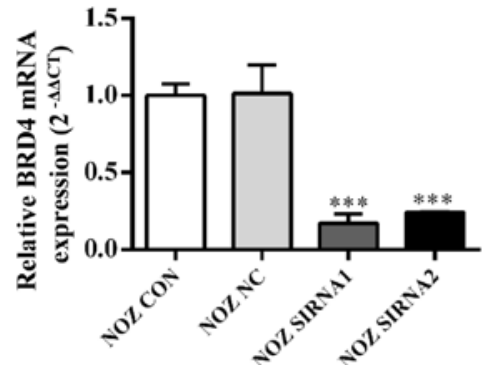

B

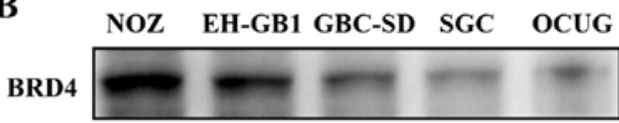

GAPDH

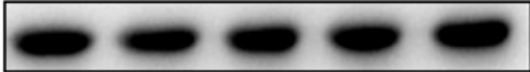

D

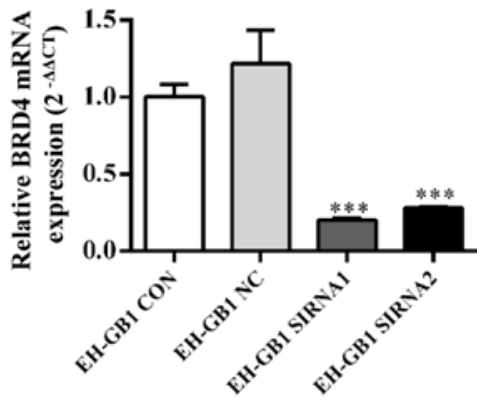

$\mathbf{F}$
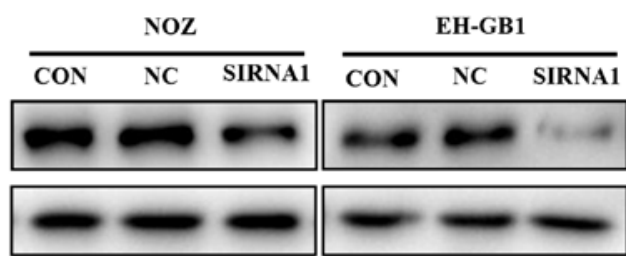

Figure 2. Expression and knockdown efficiency of BRD4 in GBC cell lines. (A and B) Expression levels of BRD4 in the GBC cell lines NOZ, EH-GB1, GBC-SD, SGC-996 and OCUG were detected by qRT-PCR and western blotting. (C) Immunofluorescence targeting BRD4 (green) and Hoechst 33342 (blue) staining in NOZ and EH-GB1 cells (x200). (D-F) Knockdown efficiency of BRD4 in NOZ and EH-GB1 cells tested by qRT-PCR and western blotting. GADPH was used as the loading control. ${ }^{* * *} \mathrm{P}<0.001$.

the Cox proportional hazards model was used for independent prognostic factors analysis. All assays were independently performed 3 times. $\mathrm{P}<0.05$ was considered to indicate a statistically significant difference.

\section{Results}

High expression of BRD4 correlates to poor prognosis of $G B C$ patients. To investigate the expression level of BRD4 in GBC tissues, we first compared BRD4 mRNA expression in 34 pairs of GBC tissues. The results showed that BRD4 expression was higher in tumor tissues than in adjacent nontumor tissues $(\mathrm{P}=0.01)$ (Fig. $1 \mathrm{~A}$ and $\mathrm{B})$. Then we evaluated BRD4 protein levels in 51 archived paraffin-embedded specimens of GBC tissues using immunohistochemistry (Fig. 1C). As shown in Fig. 1D, the BRD4 expression level was higher in tumor tissues than in their corresponding adjacent normal tissues $(\mathrm{P}<0.001)$. We further analyzed the correlation between BRD4 levels and clinicopathological data of GBC patients. The BRD4 levels were significantly related to pathologic $\mathrm{T}$ stage $(\mathrm{P}<0.001)$, lymph node metastasis $(\mathrm{P}=0.025)$ and $\mathrm{TNM}$ stage $(\mathrm{P}=0.002)$ (Table $\mathrm{I})$. The mean overall survival time of GBC patients with medium or high [4-7] BRD4 expression was 10.00 \pm 6.91 months, significantly lower than patients with negative or low [0-3] BRD4 expression $(24.67 \pm 6.72$ months, $\mathrm{P}<0.001)$ (Fig. 1E). Multivariate
Cox regression analysis indicated that BRD4 expression level was an independent prognostic factor in GBC patients $(\mathrm{P}=0.028$, Table II $)$.

BRD4 expression in GBC cell lines. Five established gallbladder cancer cell lines (NOZ, EH-GB1, GBC-SD, SGC-996, OCUG) were analyzed for expression of BRD4 by qRT-PCR and western blotting. Fig. 2A and B showed that BRD4 expression was significantly increased in GBC cell lines, particularly in the NOZ and EH-GB1 lines. Immunocytochemical staining confirmed that BRD4 protein mainly expressed on the cell nucleus of GBC cells (Fig. 2C).

Then we transfected gallbladder cancer cell lines with siRNAs against BRD4. We evaluated the efficiency of siRNA transfection by qRT-PCR and western blotting. The results showed that BRD4 mRNA and protein expression were clearly suppressed after transfection with BRD4-siRNA (Fig. 2D and E).

Effect of BRD4 on proliferation in GBC cells. To explore the role of BRD4 in GBC cell proliferation, we conducted CCK-8 and colony formation assays. Fig. 3A and B showed that the proliferative ability was inhibited in NOZ and EH-GB1 cells when silencing BRD4 $(\mathrm{P}<0.01)$. Meanwhile, downregulation of BRD4 reduced colony formation capacity of GBC cells $(\mathrm{P}<0.001)$ (Fig. 3C and D). 

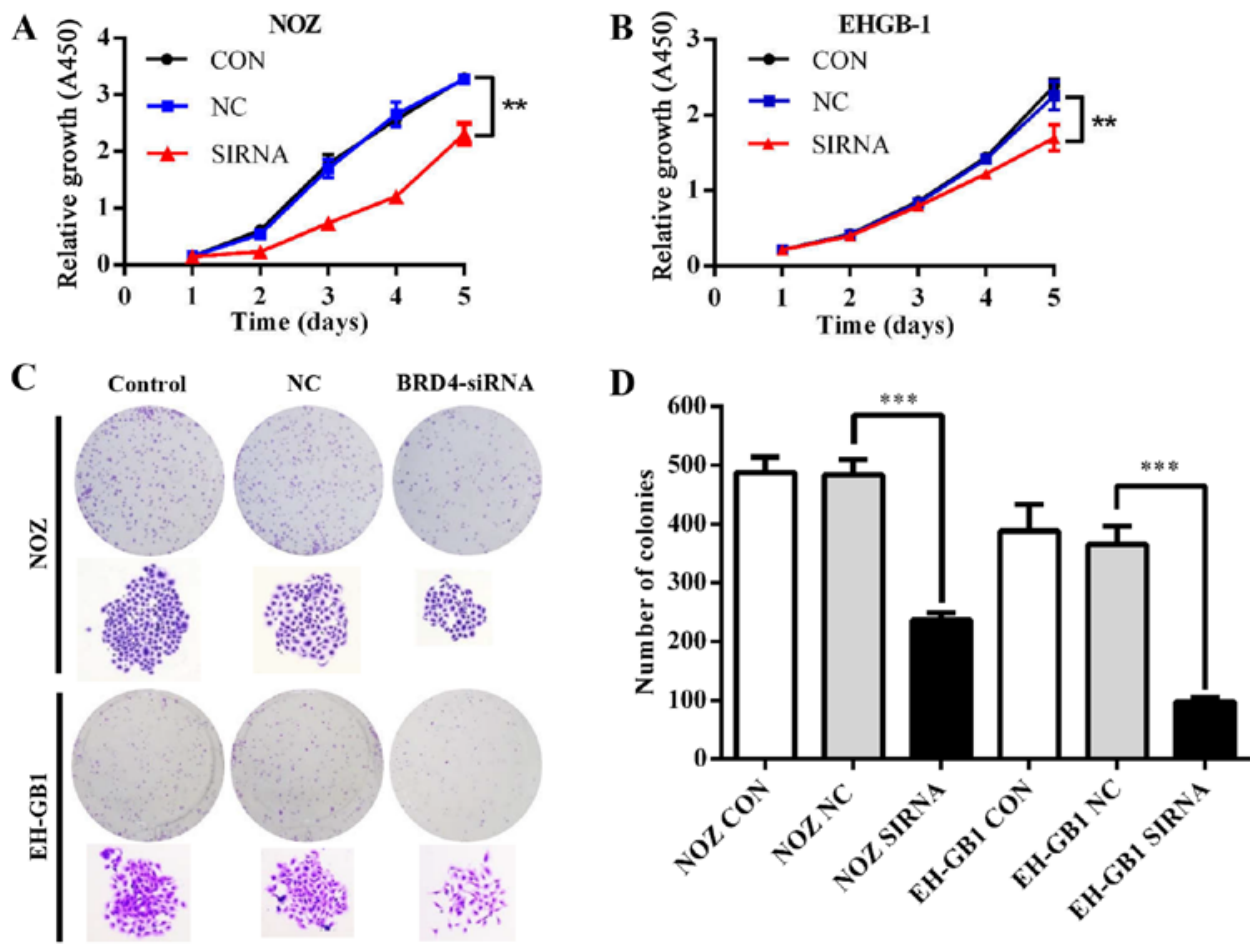

Figure 3. Effect of BRD4 silencing on GBC cell proliferation. (A and B) Cell growth curves of NOZ and EH-GB1 cells detected by the CCK-8 assay. (C) Macroscopic images of colonies formed by treated GBC cells. (D) Bar graph of the colony numbers. ${ }^{* *} \mathrm{P}<0.01,{ }^{* * *} \mathrm{P}<0.001$.

$\mathbf{A}$
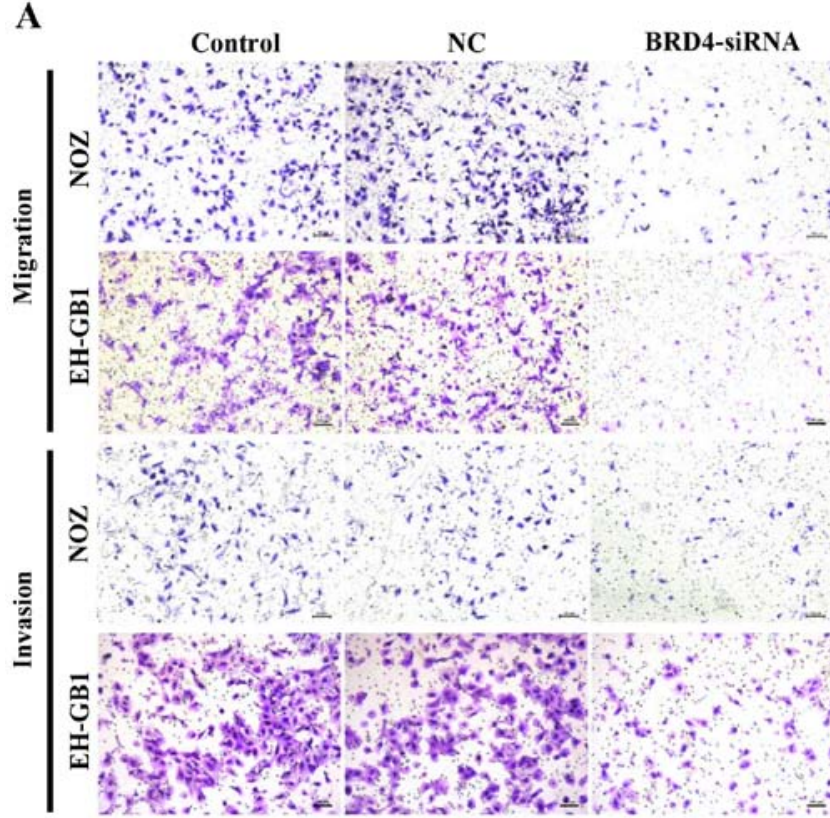

D

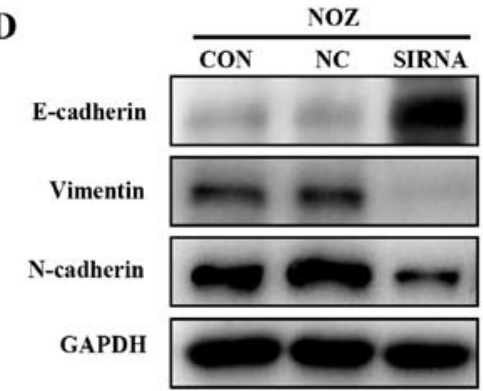

B

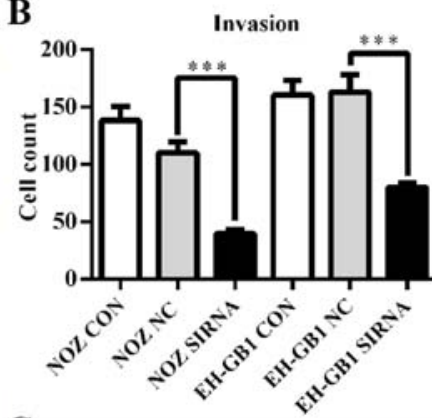

C
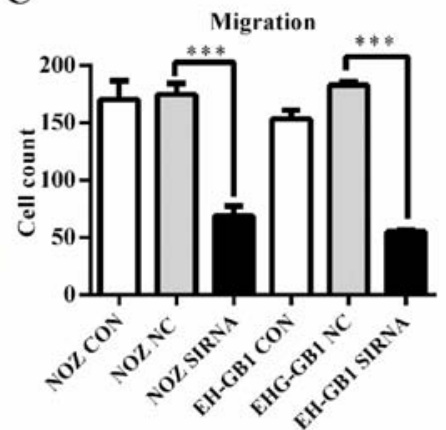

Figure 4. BRD4 silencing inhibits metastasis of GBC in vitro. (A) Transwell migration and invasion assays showed that knockdown of BRD4 inhibited GBC metastasis (scale bar, $100 \mu \mathrm{m}$ ). (B and C) Bar chart show the number of invading cells. (D) Western blotting was used to detect the expression levels of E-cadherin, N-cadherin and vimentin. ${ }^{* * *} \mathrm{P}<0.001$. 

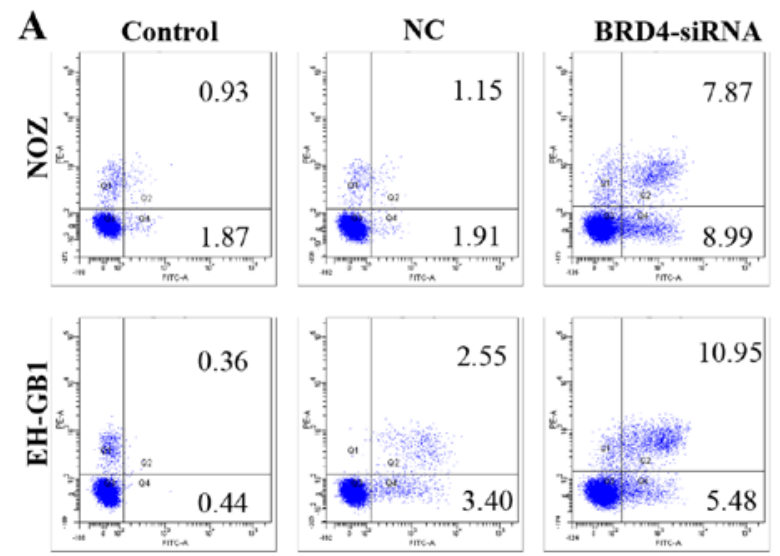

B
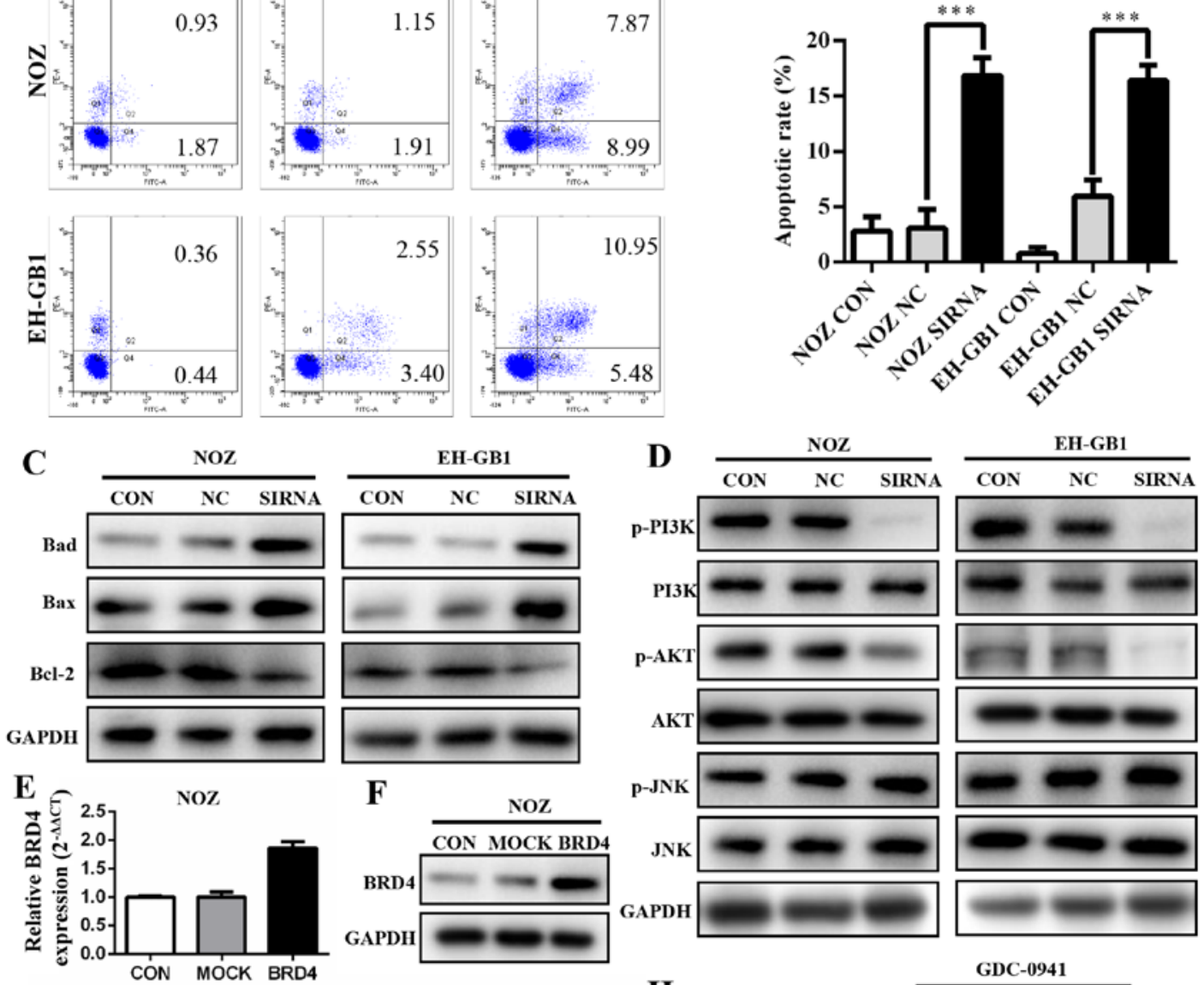

G
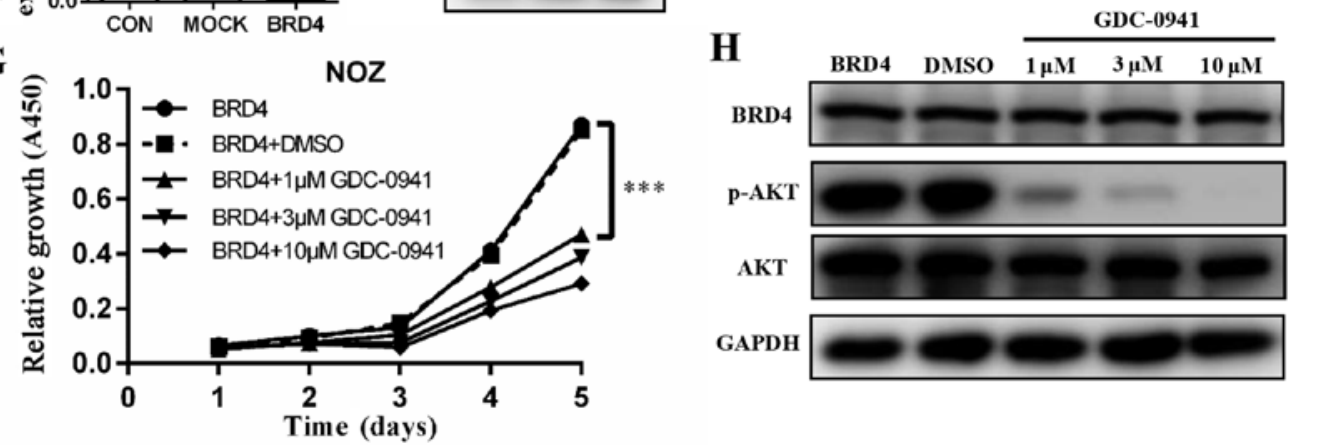

Figure 5. Effect of BRD4 silencing on GBC cell apoptosis. (A and B) Cell apoptosis was evaluated in NOZ and EH-GB1 cells by flow cytometry. Error bars represent the mean $\pm \mathrm{SD}(\mathrm{n}=3)$. (C) Western blotting was used to assess the protein expression of Bad, Bax and Bcl-2. (D) Expression levels of PI3K/AKT and JNK/SAPK pathway proteins were determined by western blotting. (E and F) qRT-PCR and western blotting demonstrated that BRD4 expression level was upregulated in NOZ cells after transfected with BRD4 full length plasmid. (G) Cell growth condition of treated NOZ cells detected by the CCK-8 assay. (H) Protein levels of BRD4, p-AKT and AKT in treated NOZ cells after $72 \mathrm{~h}$ using western blotting. ${ }^{* * *} \mathrm{P}<0.001$.

Knockdown of BRD4 inhibits GBC cell metastasis. To determine whether BRD4 deregulation affects GBC metastasis, we performed transwell assays. As shown in Fig. 4A-C, knockdown of BRD4 significantly decreased the migration and invasion abilities of GBC cells. This suggested that BRD4 could enhance the metastatic capacity of GBC. Then we examined proteins relative to epithelial-mesenchymal transition (EMT). Results revealed that when BRD4 was depleted, the expression of epithelial marker E-cadherin increased, while the expression of Vimentin and $\mathrm{N}$-cadherin (mesenchymal markers) decreased (Fig. 4D).

BRD4 silencing induces apoptosis in $G B C$ cell lines via PI3K/AKT pathway. To explore if and how BRD4 affects apoptosis, we conducted flow cytometry analysis. The results showed that apoptotic and dead cells increased significantly in the cells with BRD4 siRNA transfection $(\mathrm{P}<0.001)$. The apoptosis rates of BRD4 siRNA groups were 16.86 and $16.43 \%$ in NOZ cells and EH-GB1 cells, respectively (Fig. 5A and B). This indicated that the inhibition of BRD4 affected GBC cell apoptosis. Next, we detected the protein levels of several Bcl-2 family proteins. As shown in Fig. 5C, upregulation of Bax and Bad protein and downregulation of Bcl-2 were induced when transfected with BRD4 siRNA in GBC cells.

The PI3K/AKT and JNK/SAPK signaling pathways have been demonstrated to play essential roles in Bad regulation $(15,16)$. We found that the expression levels of p-PI3K and p-AKT decreased significantly when BRD4 was silenced. 
A
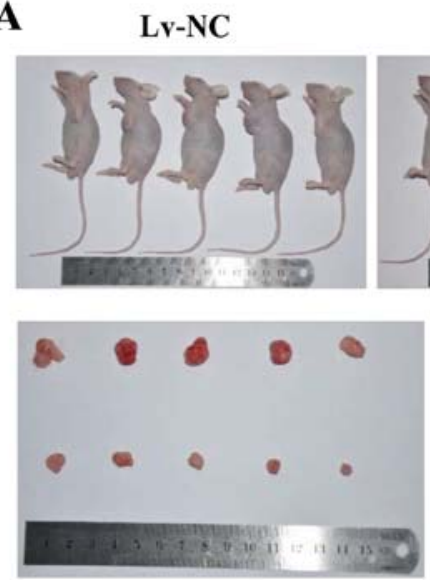

D

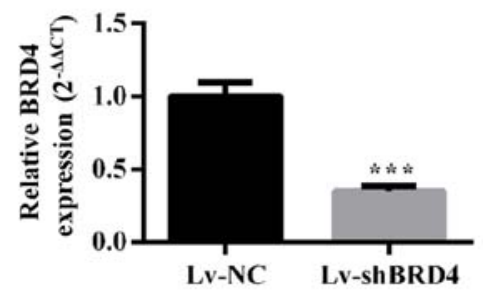

Lv-shBRD4

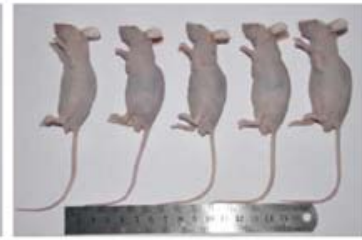

Lv-NC

Lv-shBRD4
B

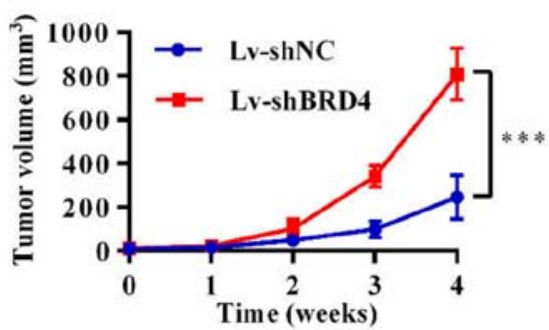

C

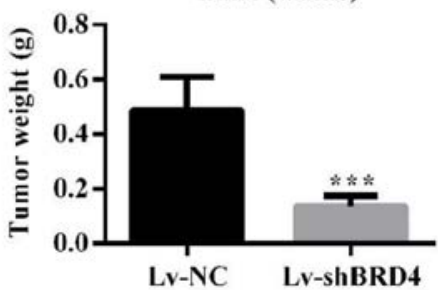

BRD4

Lv-NC Lv-shBRD4

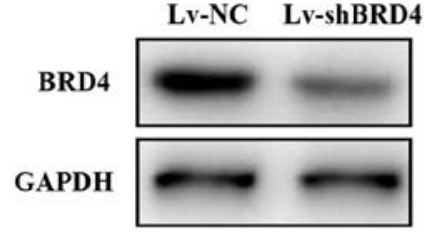

Figure 6. Knockdown of BRD4 inhibited tumor growth in nude mice. (A) Representative photos of tumour formation in nude mice injected with Lv-shBRD4 and Lv-NC cells. (B and C) The tumor volume and weight were measured in Lv-shBRD4 and Lv-NC groups. (D) Relative BRD4 mRNA and protein expression were determined using qRT-PCR and western blotting after BRD4 silencing in vivo. ${ }^{* * *} \mathrm{P}<0.001$.

Table II. Univariate and multivariate analysis for overall survival in GBC patients.

\begin{tabular}{|c|c|c|c|c|}
\hline \multirow[b]{2}{*}{ Variables } & \multicolumn{2}{|c|}{ Univariate analysis } & \multicolumn{2}{|c|}{ Multivariate analysis } \\
\hline & HR $(95 \% \mathrm{CI})$ & P-value & $\mathrm{HR}(95 \% \mathrm{CI})$ & P-value \\
\hline Sex (male/female) & $1.006(0.341-2.967)$ & 0.991 & & \\
\hline Age $(<60 / \geq 60)$ & $1.062(0.392-2.879)$ & 0.906 & & \\
\hline Histology (well + moderate/poor) & $0.801(0.187-3.427)$ & 0.765 & & \\
\hline $\mathrm{T}(\mathrm{Tis}+\mathrm{T} 1+\mathrm{T} 2 / \mathrm{T} 3+\mathrm{T} 4)$ & $2.833(1.088-7.379)$ & 0.033 & $1.217(0.396-3.743)$ & 0.732 \\
\hline $\mathrm{N}$ (absent/present) & $2.286(0.968-5.402)$ & 0.059 & & \\
\hline M (negative/ positive) & $4.012(1.410-11.411)$ & 0.009 & $2.413(0.823-7.078)$ & 0.109 \\
\hline Tumor stage (0-I/ II-IV) & $4.353(0.584-32.453)$ & 0.151 & & \\
\hline BRD4 expression (low/high) & $5.474(1.740-17.215)$ & 0.004 & $4.336(1.173-16.027)$ & 0.028 \\
\hline
\end{tabular}

HR, hazard ratio; CI, confidence interval.

However, JNK and p-JNK protein expression levels were not changed compared with controls (Fig. 5D).

To verify our findings, we transfected BRD4 full-length plasmid into NOZ cell lines, the expression level of BRD4 was markedly upregulated (Fig. 5E and F). Then cells were treated with a PI3K/AKT inhibitor (GDC-0941). The cell proliferation was inhibited (Fig. 5G) while BRD4 expression remained unchanged (Fig. 5H).

Knockdown of BRD4 inhibits tumor growth in vivo. To evaluate the effect of BRD4 on GBC growth in vivo, we constructed NOZ cell lines stably expressing shRNA-BRD4 or the negative control. Then, we injected subcutaneously Lv-shBRD4 and negative control NOZ cells into nude mice (Fig. 6A). The tumor volume and weight were significantly inhibited in the LV-shBRD4 group compared to the negative control group (Fig. 6B and C). The mRNA and protein level of BRD4 decreased in the BRD4-silenced group compared with negative control group (Fig. 6D). These results confirmed that BRD4 is essential for GBC growth regulation.

\section{Discussion}

BRD4 has been proved to possess a significant effect on cell biological activity as an important BET family member. In recent years, the effect of BRD4 has been observed in various 
types of cancer tissues and cell lines $(6,17-20)$. In this study, for the first time, we evaluated BRD4 expression in GBC tissues. We found that BRD4 was significantly upregulated in GBC tissues compared to their adjacent non-cancerous tissues. Moreover, high BRD4 expression level was correlated with poor prognosis in GBC patients. This indicates that high BRD4 protein expression may be a crucial prognostic indicator for GBC. We also demonstrated the significant suppression of GBC by downregulating the expression of BRD4 both in vitro and in vivo.

EMT has been implicated as an integral part of metastasis, and clarifying the underlying molecular mechanism of the latter is crucial for clinical therapies (21). Twist is a key member of EMT-activating transcriptional factors. Twist expression represses E-cadherin-mediated cell-cell adhesive processes and activates mesenchymal markers (22). The available evidence shows that di-acetylated Twist binds the second bromodomain of BRD4 in basal-like breast cancer (23). Another study shows that the administration of BRD4 inhibitor readily abolished TGF-induced EMT in small cell lung cancer (21). In this study, knockdown of BRD4 in GBC cells enhanced protein expression of the epithelial marker E-cadherin and reduced the protein expression of mesenchymal markers $\mathrm{N}$-cadherin and vimentin. Our data suggest that BRD4 downregulation inhibits migration and invasion of GBC cells by inducing EMT.

The initiation of apoptosis results in cell death. Bcl-2 family which includes pro- and anti-apoptotic molecules act as regulatory protein $(24,25)$. All the BH3-only proteins, which include Bcl-2-associated death promoter (Bad), can activate Bcl-2-associated $\mathrm{X}$ protein (Bax) indirectly by binding to and inhibiting the BCL-2 (26). Our data further supported that cancer cell death occurred due to decreased expression of $\mathrm{Bcl}-2$ and increased expression of $\mathrm{Bad}$ and $\mathrm{Bax}$ in BRD4 depleted GBC cells. The previous study indicates that PI3K/AKT is involved in Bad inactivation, whereas the JNK pathway is associated with Bad activation (27). In our study, depletion of BRD4 inhibited the expression of phosphorylation AKT, but we did not find expression change for JNK. Moreover, BRD4 expression level remained unchanged after using PI3K/AKT inhibitor, which confirmed that downregulation of BRD4 in GBC cells induced apoptosis via PI3K/AKT pathway.

In summary, we demonstrated that BRD4 deregulation is involved in GBC progression. Knockdown of BRD4 could effectively affect cell proliferation, metastasis and apoptosis in GBC cell lines and inhibit tumor growth in vivo. We hypothesize that BRD4 plays an essential role in GBC apoptosis by downregulating phosphorylation levels of AKT via the PI3K/AKT pathway. Inhibition of BRD4 expression may be a novel therapeutic strategy for GBC. Therefore, BRD4 may be a novel promising prognostic marker and an antitumor target in the treatment of GBC.

\section{Acknowledgements}

This study was supported by the National Natural Science Foundation of China (no. 31501127), Shanghai Sailing Program (no. 16YF1407100), Project Funded by China Postdoctoral Science Foundation (no. 2015M571577), and Nanjing Medical
University Science and Technology Development Fund (no. 2016NJMU132).

\section{References}

1. Garg PK, Pandey D and Sharma J: The surgical management of gallbladder cancer. Expert Rev Gastroenterol Hepatol 9: 155-166, 2015.

2. Müller BG, De Aretxabala X and González Domingo M: A review of recent data in the treatment of gallbladder cancer: what we know, what we do, and what should be done. Am Soc Clin Oncol Educ Book e165-e170, 2014. doi: 10.14694/EdBook_ AM.2014.34.e165.

3. Lu W, Hu Y, Ma Q, Zhou L, Jiang L, Li Z, Zhao S, Xu Y, Shi W, Li S, et al: miR-223 increases gallbladder cancer cell sensitivity to docetaxel by downregulating STMN1. Oncotarget 7: 62364-62376, 2016.

4. Dhalluin C, Carlson JE, Zeng L, He C, Aggarwal AK and Zhou MM: Structure and ligand of a histone acetyltransferase bromodomain. Nature 399: 491-496, 1999.

5. Hu Y, Zhou J, Ye F, Xiong H, Peng L, Zheng Z, Xu F, Cui M, Wei C, Wang X, et al: BRD4 inhibitor inhibits colorectal cancer growth and metastasis. Int J Mol Sci 16: 1928-1948, 2015.

6. Andrieu G, Tran AH, Strissel KJ and Denis GV: BRD4 regulates breast cancer dissemination through Jagged1/Notch1 signaling. Cancer Res 76: 6555-6567, 2016.

7. Dey A, Chitsaz F, Abbasi A, Misteli T and Ozato K: The double bromodomain protein Brd4 binds to acetylated chromatin during interphase and mitosis. Proc Natl Acad Sci USA 100: 8758-8763, 2003.

8. Shahbazi J, Liu PY, Atmadibrata B, Bradner JE, Marshall GM, Lock RB and Liu T: The bromodomain inhibitor JQ1 and the histone deacetylase inhibitor panobinostat synergistically reduce $\mathrm{N}-\mathrm{Myc}$ expression and induce anticancer effects. Clin Cancer Res 22: 2534-2544, 2016.

9. Wu SY, Lee AY, Lai HT, Zhang H and Chiang CM: Phospho switch triggers Brd4 chromatin binding and activator recruitment for gene-specific targeting. Mol Cell 49: 843-857, 2013.

10. Marcotte R, Sayad A, Brown KR, Sanchez-Garcia F, Reimand J, Haider M, Virtanen C, Bradner JE, Bader GD, Mills GB, et al: Functional genomic landscape of human breast cancer drivers, vulnerabilities, and resistance. Cell 164: 293-309, 2016.

11. Sahai V, Kumar K, Knab LM, Chow CR, Raza SS, Bentrem DJ, Ebine K and Munshi HG: BET bromodomain inhibitors block growth of pancreatic cancer cells in three-dimensional collagen. Mol Cancer Ther 13: 1907-1917, 2014.

12. Liao YF, Wu YB, Long X, Zhu SQ, Jin C, Xu JJ and Ding JY: High level of BRD4 promotes non-small cell lung cancer progression. Oncotarget 7: 9491-9500, 2016.

13. Meseure D, Vacher S, Alsibai KD, Nicolas A, Chemlali W, Caly M, Lidereau R, Pasmant E, Callens C and Bieche I: Expression of ANRIL-polycomb complexes-CDKN2A/B/ARF genes in breast tumors: Identification of a two-gene (EZH2/CBX7) signature with independent prognostic value. Mol Cancer Res 14: 623-633, 2016.

14. Li Z, Chen Y, Wang X, Zhang H, Zhang Y, Gao Y, Weng M, Wang L, Liang H, Li M, et al: LASP-1 induces proliferation, metastasis and cell cycle arrest at the $\mathrm{G} 2 / \mathrm{M}$ phase in gallbladder cancer by down-regulating S100P via the PI3K/AKT pathway. Cancer Lett 372: 239-250, 2016.

15. Bhakar AL, Howell JL, Paul CE, Salehi AH, Becker EB, Said F, Bonni A and Barker PA: Apoptosis induced by p75NTR overexpression requires Jun kinase-dependent phosphorylation of Bad. J Neurosci 23: 11373-11381, 2003.

16. Datta SR, Dudek H, Tao X, Masters S, Fu H, Gotoh Y and Greenberg ME: Akt phosphorylation of BAD couples survival signals to the cell-intrinsic death machinery. Cell 91: 231-241, 1997.

17. Segura MF, Fontanals-Cirera B, Gaziel-Sovran A, Guijarro MV, Hanniford D, Zhang G, González-Gomez P, Morante M, Jubierre L, Zhang W, et al: BRD4 sustains melanoma proliferation and represents a new target for epigenetic therapy. Cancer Res 73: 6264-6276, 2013.

18. Shimamura T, Chen Z, Soucheray M, Carretero J, Kikuchi E, Tchaicha JH, Gao Y, Cheng KA, Cohoon TJ, Qi J, et al: Efficacy of BET bromodomain inhibition in Kras-mutant non-small cell lung cancer. Clin Cancer Res 19: 6183-6192, 2013. 
19. Tögel L, Nightingale $R$, Chueh AC, Jayachandran A, Tran H, Phesse T, Wu R, Sieber OM, Arango D, Dhillon AS, et al: Dual targeting of bromodomain and extraterminal domain proteins, and WNT or MAPK signaling, inhibits c-MYC expression and proliferation of colorectal cancer cells. Mol Cancer Ther 15: 1217-1226, 2016.

20. Wu X, Liu D, Tao D, Xiang W, Xiao X, Wang M, Wang L, Luo G, Li Y, Zeng F, et al: BRD4 regulates EZH2 transcription through upregulation of C-MYC and represents a novel therapeutic target in bladder cancer. Mol Cancer Ther 15: 1029-1042, 2016.

21. Chang H, Liu Y, Xue M, Liu H, Du S, Zhang L and Wang P. Synergistic action of master transcription factors controls epithelial-to-mesenchymal transition. Nucleic Acids Res 44: 2514-2527, 2016.

22. Yang J, Mani SA, Donaher JL, Ramaswamy S, Itzykson RA, Come C, Savagner P, Gitelman I, Richardson A and Weinberg RA: Twist, a master regulator of morphogenesis, plays an essential role in tumor metastasis. Cell 117: 927-939, 2004.
23. Shi J, Wang Y, Zeng L, Wu Y, Deng J, Zhang Q, Lin Y, Li J, Kang T, Tao M, et al: Disrupting the interaction of BRD4 with diacetylated Twist suppresses tumorigenesis in basal-like breast cancer. Cancer Cell 25: 210-225, 2014.

24. Hanahan D and Weinberg RA: Hallmarks of cancer: The next generation. Cell 144: 646-674, 2011

25. Zhang H, Li Z, Chu B, Zhang F, Zhang Y, Ke F, Chen Y, Xu Y, Liu S, Zhao S, et al: Upregulated LASP-1 correlates with a malignant phenotype and its potential therapeutic role in human cholangiocarcinoma. Tumour Biol 37: 8305-8315, 2016.

26. Delbridge AR and Strasser A: The BCL-2 protein family, BH3-mimetics and cancer therapy. Cell Death Differ 22: 1071-1080, 2015

27. Kamada H, Nito C, Endo $\mathrm{H}$ and $\mathrm{Chan} \mathrm{PH}$ : Bad as a converging signaling molecule between survival PI3-K/Akt and death JNK in neurons after transient focal cerebral ischemia in rats. J Cereb Blood Flow Metab 27: 521-533, 2007. 